\title{
Тенденции развития методов и устройств лазерной литографии для синтеза элементов дифракционной оптики
}

\author{
В.П. Корольков ${ }^{*}$, А.Г. Седухин, В.Н. Хомутов, Р.В. Шиманский, А.Е. Качкин \\ Институт автоматики и электрометрии СО РАН \\ *E-mail: victork@iae.nsk.su
}

DOI:10.31868/RFL2018.227-229

За последнее время компьютерно-синтезированные дифракционные оптические элементы (КСДОЭ) стали находить все большее практическое применение в оптических системах, дополняя традиционные рефракционные и отражательные оптические элементы. Активно разрабатываются и исследуются КСДОЭ с тонкой субволновой рельефно-фазовой структурой, в которых изменение фазы волны осуществляется не за счет изменения оптической толщины выступов и впадин структуры, а за счет изменения так называемой геометрической фазы (фазы Панчаратнама-Берри) при локальном (субволновом) изменении состояния поперечной поляризации оптической волны. Такие структуры часто относят не к КСДОЭ, а к новым тонким метаструктурам с управляемым лазерно-индуцированным двулучепреломлением в их субволновых зонах. В данной статье мы будем называть последние элементы КСДОЭ с изменением геометрической фазы волны или кратко ДОЭИГФ. К их достоинствам можно отнести то, что они имеют световую эффективность близкую к $100 \%$ и способность работы в широком интервале длин волн (в белом свете). Потенциально, ДОЭИГФ могут заменить традиционные толстые оптические элементы. Вместе с тем, следует отметить, что сама рельефно-фазовая структура ДОЭИГФ, является существенно более сложной, чем у традиционных КСДОЭ и требует применения новых тонкопленочных оптических материалов и новых высокоразрешающих технологий записи самих структур, с продвижением в область все меньших и меньших размерных масштабов. Более конкретно, - ДОЭИГФ изготавливаются, в настоящее время, в основном по достаточно дорогой технологии высокоразрешающей электронно-лучевой нанолитографии. Соответственно, относительно большие время и стоимость технологии изготовления данных элементов являются сдерживающими факторами для их дальнейшего исследования и более широкого практического применения.

Помимо указанных субволновых ДОЭИГФ, в настоящее время востребованными на практике являются также субволновые элементы, выполняющие функции антиотражательных и поляризационно-конвертирующих элементов, а также элементов, преобразующих форму лазерного пучка (заметим, что все эти функции способны выполнять и новые указанные элементы). Такие элементы, как правило, должны иметь глубокий фазовый профиль их структуры, что требует применения особых оптических материалов и технологии их изготовления. Кроме того, к традиционным КСДОЭ, в последнее время предъявляются требования по их более высокой топологической (абсолютной) точности, что также обычно сопряжено с требованием уменьшения размера сфокусированного лазерного пучка.

Резюмируя, можно сказать, что для изготовления современных КСДОЭ, актуальным является дальнейшее повышение разрешающей способности и точности технологий их изготовления, наряду с использованием новых материалов для записи. В настоящее время существует две основные группы компьютерных технологий изготовления субволновых КСДОЭ - электронно-лучевая и лазерная. Потенциально 
разрешающая способность электронно-лучевой технологии выше чем для лазерной записи на один - полтора порядка (5-20 нм против 400-800 нм). Но в случае необходимости использования толстого слоя резиста, эта разница почти нивелируется из-за рассеяния электронов в резисте. Технологии прямой лазерной записи более консервативны в силу существенно меньшего, на сегодня, рынка сбыта. Поэтому потенциал технических возможностей современных технологий и установок прямой лазерной записи для записи КСДОЭ далеко не реализован и здесь можно ожидать существенного прогресса в случае приближения их разрешающей способности к показателям электронно-лучевой нанолитографии для тонкопленочных процессов.

В данной работе сделан акцент на развитие методов и устройств лазерной нанолитографии оптического диапазона длин волн. Речь идет о возможности реализации лазерной записи с разрешением не хуже 100 нм, что вполне достаточно для многих задач синтеза субволновых структур для оптического диапазона. С учетом вышеуказанных современных требований к субволновым КСДОЭ и применительно к использованию и дальнейшему развитию термохимической технологии прямой лазерной записи КСДОЭ, в работе обсуждаются способы решения следующих задачи:

- исследование тонких металлических пленок, в качестве материала записи, для получения высокого контраста записи при малой толщине пленок (в частности, пленок титана, циркония и хрома),

- использование новых нелинейных сверхразрешающих механизмов записи, включая режим сквозного окисления и, соответственно, просветления облучаемых пленок,

- уменьшение рабочей длины волны технологического лазера (в частности до ГУФ длины волны 266 нм), а также увеличение показателя преломления в зоне фокусировки (до 1,4), при записи в твердых и жидких средах

- преодоление дифракционного предела по размеру сфокусированного лазерного пятна при дальнепольной (на расстоянии 0,2 - 1 мм) фокусировке.

Для преодоления дифракционного предела по размеру сфокусированного лазерного пятна предполагается использование следующих методов:

- применение фокусирующей оптики с кольцевой формой апертуры, а также поиск и применение методов дополнительного «обострения» фокусировки, за счет специального перераспределения интенсивности света на апертуре фокусирующей оптики и аппаратного обострения так называемой функции аподизации,

- поиск и применение новых и уже традиционных видов лазерных пучков с неоднородным распределением поляризации по апертуре фокусирующей оптики,

- поиск и применение методов пространственно-частотной фильтрации лазерного пучка (фильтрации Торальдо).

Как показывают проведенные теоретические исследования [], применение совокупности указанных методов по преодолению дифракционного предела, а также решение задачи по возможности работы на длине волны 266 нм, позволяет получить размеры лазерного пятна в дальней зоне (с рабочим отрезком объектива порядка 1 мм) и по половинному уровню интенсивности (от максимальной в центральной точке сфокусированного пятна) 105 и 70 нм, соответственно, при записи в воздушной среде и в средах с показателем преломления 1,5. Вместе с тем, форма распределения интенсивности света в фокальной области (функция рассеяния точки) оказывается близка к возведенной в квадрат функции Бесселя первого рода и нулевого порядка. То есть сфокусированный пучок близок к идеальному пучку Бесселя с относительно высоким уровнем побочных максимумов и относительно низкой эффективностью в полезном центральном лепестке. Это обстоятельство необходимо согласовать далее с вышеуказанным выбором материалов, режимов, а также соответствующих тепловых расчетов при термохимической записи. 
Приведенные методы фокусировки лазерного пучка требуют их адекватной аппаратной реализации. В этой связи в докладе также обсуждаются направления и промежуточные результаты разработки установок лазерной записи.

\section{Литература}

[1] Sedukhin A.G et al, Opt. Commun. Vol. 407, pp. 217-226 (2018) 